\title{
La représentation du personnage féminin dans le manuel d'Anglais en Algérie : vers une éclipse totale?
}

Férida Lakhdar-Barka

\section{(Q) OpenEdition}

\section{Journals}

Édition électronique

URL : http://journals.openedition.org/trema/2634

DOI : $10.4000 /$ trema.2634

ISSN : 2107-0997

Éditeur

Faculté d'Éducation de l'université de Montpellier

Édition imprimée

Date de publication : 1 décembre 2011

Pagination : $114-127$

ISSN : 1167-315X

Référence électronique

Férida Lakhdar-Barka, « La représentation du personnage féminin dans le manuel d'Anglais en Algérie : vers une éclipse totale? », Tréma [En ligne], 35 - 36 | 2011, mis en ligne le 01 décembre 2013, consulté le 19 avril 2019. URL : http://journals.openedition.org/trema/2634 ; DOI : 10.4000/trema.2634

Ce document a été généré automatiquement le 19 avril 2019.

Trema 
La représentation du personnage féminin dans le manuel d'Anglais en Algérie : vers une éclipse totale?

Férida Lakhdar-Barka

I. Introduction 
1 La réforme du système éducatif algérien, initiée en $2000^{1}$, a eu pour effet une refonte totale des programmes, suivie de l'édition d'une nouvelle génération de manuels scolaires ${ }^{2}$. Cette réforme tant attendue se propose, selon ses concepteurs, de répondre à de nouveaux besoins à la fois d'ordre socioculturel, économique et politique. À titre d'exemple, l'enseignement des langues étrangères a pour objectif de «doter l'apprenant des atouts indispensables pour réussir dans le monde de demain" " ${ }^{3}$. "Réussir" semble être le mot-clé d'un programme jugé dans l'ensemble assez ambitieux. Mais, quelques années après son application, "seuls $20 \%$ des Algériens jugent que le niveau de l'école est bon " ${ }^{4}$ et le niveau académique à l'université (qui subit l'évolution du niveau scolaire) est en baisse constante.

Dès le début des années 2000 , le ministère

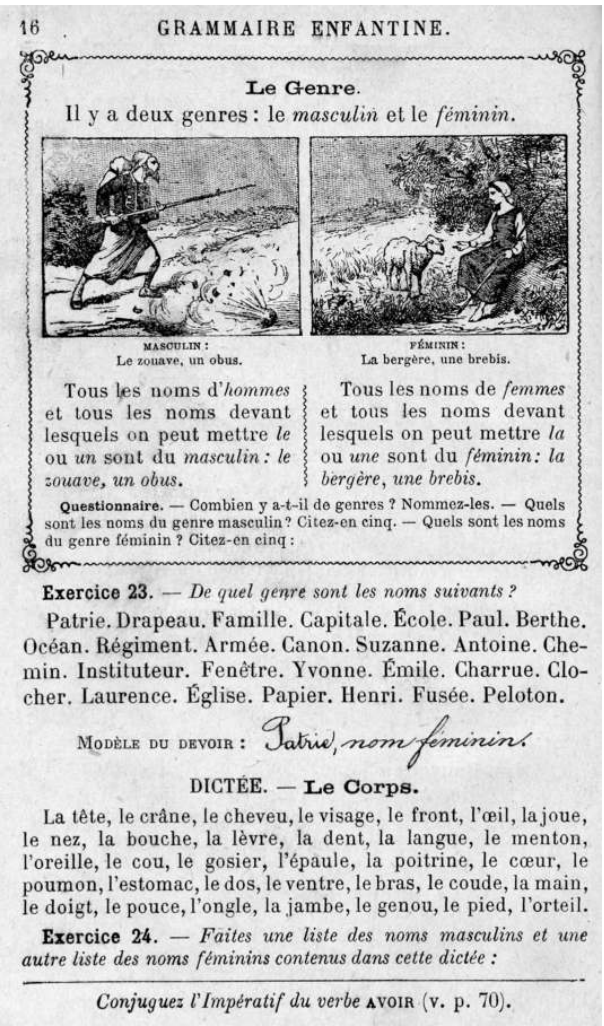
de l'Enseignement supérieur et de la recherche scientifique (MESRS) a encouragé la mise en place de laboratoires de recherche au sein des universités algériennes afin de remédier à cette situation. Le Laboratoire pour la création d'outils d'apprentissage en langues étrangères créé par arrêté ministériel en 2000 et rattaché à la Faculté des lettres, langues et arts de l'université d'Oran (LOAPL), dont nous sommes membre depuis sa création, a pour objectif de concevoir et d'éditer des supports didactiques en langues étrangères. Parmi ses projets, un programme de recherche-action a été initié en 2003, visant en priorité la confection de manuels d'apprentissage pour la licence d'Anglais ${ }^{5}$, et à long terme, l'élaboration d'un modèle didactique pour l'enseignement de l'Anglais au niveau du secondaire. La phase initiale de ce dernier objectif consiste à dresser un état des lieux des manuels utilisés au lycée, dont fait partie la présente analyse diachronique ${ }^{6}$ des représentations sexuées, à partir des manuels de 3e année secondaire (Terminale) édités entre 1987 et 2007.

Nous présentons dans le cadre de cet article les données quantitatives et qualitatives concernant la présence des personnages féminins qui révèlent un décalage entre le discours didactique et la réalité sociale, en même temps qu'un écart entre le curriculum formel et le curriculum réel (PERRENOUD, 1993). Le traitement discriminatoire du personnage féminin au sein des manuels scolaires a fait l'objet de nombreux travaux dans plusieurs pays, (BOUCHOUCHA et LOCOH, 2008 ; BRUGEILLES, CROMER et LOCOH, 2008 ; HERRERA, 2004 ; JUNOD, 2001 ; PAIVANDI, 1998 ; RHIWI, 1999), mais en Algérie, peu de travaux ont abordé cette question ${ }^{7}$ qui, comparée à d'autres aspects problématiques concernant l'école, paraît secondaire. Pourtant, pour comprendre les raisons du dysfonctionnement du système éducatif, on ne peut ignorer l'importance de l'impact du manuel sur l'apprenant, surtout si celui-ci constitue le seul accès au savoir et à la culture écrite ( ACHOUR et MORSLY, 1985, p.10). 
4 Nous présenterons la méthodologie choisie et le corpus, puis seront exposées l'interprétation des données et leur discussion; enfin, la conclusion présentera quelques propositions de modification dans la conception des manuels scolaires, dans la perspective de changement propre à la recherche-action.

\section{Une recherche qualitative et quantitative orientée vers le changement}

5 Le contexte professionnel, le parcours du chercheur, ainsi que la nature de l'objet de recherche déterminent le type d'approche à entreprendre ${ }^{8}$. La recherche-action qui est «surtout orientée vers le changement et permet les relations entre le chercheur et les acteurs, laissant une place à la subjectivité et à son expression » (PINARD et al., 2004, p.60) a semblé appropriée, d'autant qu'elle privilégie la méthode qualitative. Il a paru nécessaire d'y associer la méthode quantitative pour mesurer la visibilité du personnage féminin dans les parties textuelles et iconographiques des manuels concernés. Comme l'affirme FRY $(1981$, p.146) : « ...the merging of qualitative and quantitative techniques in a single study or design...would seem to offer excellent possibilities for developing a deeper understanding of educational processes ${ }^{9}{ }^{9}$.

BRUGEILLES et al., (2008) mentionnent le risque de biais méthodologique guettant le chercheur qui, occupé à débusquer les éléments discriminatoires apparents sachant que les stéréotypes les plus criants peuvent avoir disparu (cf. OUESLATI et al., 2010, p.22), peut ne pas relever une discrimination "en creux». Pour limiter ce risque, les auteures proposent de "décrire le système de genre véhiculé » en s'efforçant de "décrypter la construction du sexe social» à travers l'ensemble des caractéristiques du personnage. Évoquant les travaux de VAN DER MAREN (1996) et de MUCCHIELLI (1988), RICHARD mentionne également deux sortes de biais : «Il s'agit des biais affectifs et des biais idéologiques, les premiers étant directement liés aux émotions et à la sensibilité du chercheur alors que les seconds sont en lien avec son idéologie et sa théorie a priori » (2006, p.185). Dans ce cas aussi, l'utilisation de grilles de lecture et de procédés de validation des instruments d'analyse demeurent indispensables. Un dernier point parmi les limites de cette recherche concerne le fait que les résultats quantitatifs et qualitatifs ne renseignent pas sur ce qui se passe en classe (MORIN, 2004), c'est-à-dire sur les apprentissages, les attitudes et représentations des élèves (OUESLATI et al., 2010) ainsi que ceux des enseignants.

\section{Une étude diachronique de cinq manuels d'anglais datant de 1987 à 2007}

7 Le corpus comprend cinq manuels, approuvés par le ministère de l'Éducation nationale, tous édités par des organismes étatiques (l'Institut pédagogique national remplacé plus tard par l'office national des publications scolaires), à l'exception de celui de 2003 publié par une maison d'édition privée (Éditions IBN KHALDOUN ou EDIK). Les cinq manuels s'adressent au niveau de la 3e année secondaire (Terminale) toutes filières confondues et sont confectionnés par une équipe dirigée par un inspecteur de l'enseignement secondaire, exception faite de l'ouvrage de 2007 (post-réforme) rédigé par des universitaires. Il est à noter que l'ouvrage de 2003 n'est pas utilisé comme un support pédagogique officiel par les enseignants, mais plutôt comme un appoint ${ }^{10}$. 
8 Les bornes chronologiques (1987-2007) constituent des repères importants, tant du point de vue de l'histoire du pays ${ }^{11}$ que de l'évolution du système éducatif ${ }^{12}$. Durant ces deux décennies, l'Algérie a connu deux périodes critiques. La première décennie était caractérisée par la violence terroriste et a démarré quelque temps après les émeutes de 1988, et la deuxième décennie a vu l'accélération de la mondialisation et ses bouleversements dans les différents domaines.

\section{Une occultation de la représentation féminine en décalage avec la réalité sociale}

9 L'analyse quantitative mesure la visibilité du personnage féminin. La notion de personnage féminin renvoie à un personnage fictif construit à partir d'un ensemble de caractéristiques qui le différencient du personnage masculin sur le plan du sexe social. Est quantifié le nombre de documents où apparaît le personnage féminin par rapport au total des documents iconographiques (photographies ou dessins) et des documents textuels (représentés par les textes exemplificateurs, excluant ainsi les textes déclencheurs et analytiques $)^{13}$.

Tableau 1 : Nombre de documents iconographiques et textuels des cinq manuels, où figurent des personnages féminins, des personnages masculins et des personnages des deux sexes

\begin{tabular}{|c|c|c|c|c|c|c|c|c|}
\hline Manuels & \multicolumn{2}{|c|}{ NOMBRE DE DOCUMENTS ICONOGRAPHIQUES } & \multicolumn{3}{|c|}{ NOMBRE DE DOCUMENTS TEXTUELS } \\
\hline & $\begin{array}{c}\text { Avec un } \\
\text { personnage } \\
\text { féminin }\end{array}$ & $\begin{array}{c}\text { Avec un } \\
\text { personnage } \\
\text { masculin }\end{array}$ & $\begin{array}{c}\text { Avec des } \\
\text { personnages } \\
\text { des 2 sexes }\end{array}$ & $\begin{array}{c}\text { Total des } \\
\text { documents } \\
\text { icono- } \\
\text { graphiques }\end{array}$ & $\begin{array}{c}\text { Avec un } \\
\text { personnage } \\
\text { féminin }\end{array}$ & $\begin{array}{c}\text { Avec un } \\
\text { personnage } \\
\text { masculin }\end{array}$ & $\begin{array}{c}\text { Avec des } \\
\text { personnages } \\
\text { des 2 sexes }\end{array}$ & $\begin{array}{c}\text { Total } \\
\text { des documents } \\
\text { textuels }\end{array}$ \\
\hline 1987 & 9 & 27 & 19 & 82 & 11 & 45 & 18 & 153 \\
\hline 2000 & & & & 0 & 9 & 55 & 10 & 78 \\
\hline 2001 & 12 & 65 & 3 & 80 & 4 & 33 & 26 & 103 \\
\hline 2003 & 25 & 61 & 6 & 160 & 28 & 53 & 7 & 150 \\
\hline 2007 & 5 & 25 & 24 & 81 & 6 & 56 & 15 & 102 \\
\hline Total & $\mathbf{5 1}$ & $\mathbf{1 7 8}$ & $\mathbf{5 2}$ & $\mathbf{4 0 2}$ & $\mathbf{5 8}$ & $\mathbf{2 4 2}$ & $\mathbf{7 6}$ & $\mathbf{5 8 6}$ \\
\hline
\end{tabular}

Le premier constat est qu'un nombre important de documents textuels ou iconographiques ne comporte pas de personnages sexués. Le deuxième constat est que les documents - iconographiques et surtout textuels - comportent davantage de personnages masculins que de féminins. Il y a environ trois fois plus d'images et quatre fois plus de textes avec un personnage masculin. Dans les images, comme dans les textes, la présence des femmes est moindre en 2007, alors qu'au moins dans les textes, la présence des hommes s'est maintenue. Par exemple, dans les documents iconographiques, cette proportion 3 fois plus faible que celle des hommes, en 1987, devient 5 fois plus faible en 2007.

11 L'inégale présence de la figure féminine par rapport à la figure masculine est également d'ordre qualitatif, du point de vue de la diversité des situations sociales qui pourraient lui conférer une "épaisseur » ou un rôle dans l'environnement où elle évolue, que ce soit dans la sphère privée ou publique. À titre d'exemple, en poursuivant la comparaison du manuel de 1987 avec celui de 2007, dans l'ensemble des documents du premier ouvrage, la femme est décrite en tant que personne active, libre et responsable, que ce soit dans le domaine du travail, du sport, de l'éducation, de son implication dans la lutte pour la préservation de l'environnement ou dans la politique. Elle partage l'espace avec son 
homologue masculin dans les contextes publics et privés. De plus, le concepteur du manuel a inclus parmi les onze documents textuels six textes écrits par des auteures. Tous les sujets ont un lien avec le réel et le quotidien de l'apprenant. Par contre, dans le manuel de 2007, la majorité des documents privilégie des personnages célèbres, soit appartenant à la mythologie grecque (Hélène, Pénélope, Urania), soit à une réalité éloignée de celle des utilisateur-trices de ce manuel ${ }^{14}$ ), telles que la princesse Diana, l'actrice algérienne Biyouna, la scientifique Marie cURIE, l'ex-groupe de chanteuses Abba ou l'actrice américaine Cindy CRAWFORD vantant un produit sur une affiche de publicité. Parmi les six documents textuels représentant un personnage féminin, deux sont écrits par des femmes dont un adapté (réécrit) par un homme.

12 Si l'on s'intéresse cette fois non pas au contenu des manuels, mais au paratexte, qui informe sur la place des femmes dans le processus de confection des manuels, on constate tout d'abord, que les concepteurs des cinq manuels sont tous des hommes (quatre inspecteurs de l'enseignement secondaire pour les quatre premiers ouvrages et une équipe d'universitaires pour le dernier). Viennent ensuite les équipes rédactionnelles qui sont diversement constituées : celle de l'ouvrage de 1987 est formée de cinq femmes, celle du manuel de 2000 de deux hommes; le manuel de 2001 a été entièrement conçu par l'inspecteur lui-même ; l'ouvrage de 2003 ne comporte pas d'équipe rédactionnelle mais adresse des remerciements à plusieurs femmes (collègues et personnels d'édition) dont l'épouse du concepteur, et celui de 2007 est co-écrit par trois hommes, mais comporte également des remerciements à l'égard du personnel enseignant féminin. Il est utile d'ajouter que le manuel de 1987 est le seul qui comporte un chapitre (sur les neuf) entièrement consacré aux femmes.

Pourquoi aujourd'hui le personnage féminin est-il moins visible dans les manuels scolaires qu'auparavant, alors que les femmes ont investi l'espace public d'une façon indéniable et que les statistiques le prouvent? Ainsi le taux de scolarisation des filles et les chiffres concernant l'emploi féminin ont augmenté de façon régulière. Entre 1987 et 2007 (bornes chronologiques de notre étude), le taux de scolarisation des filles inscrites en 1re année primaire est passé de $87,8 \%$ à $95 \%$, et celui concernant l'emploi féminin (population active comprise entre 15 et 50 ans) a grimpé de 5,4\% à 14,7\%. (Office national des statistiques, Algérie). De surcroit, il est à noter qu'un grand nombre de femmes exercent des activités non déclarées, à domicile ou dans des entreprises privées et que, par conséquent elles ne sont pas quantifiées (SADOU, 2007).

De plus, si l'on compare les orientations officielles (qui prônent l'égalité face au savoir, l'intégration des connaissances dans des projets liés au vécu des apprenants et l'ouverture au monde moderne) ${ }^{15}$ avec le véritable message qui sous-tend ces manuels, on décèle un écart certain.

\section{Une visibilité ambiguë}

Pour autant, le taux de visibilité n'est pas toujours synonyme de valorisation.

Par exemple, si l'on compare les manuels de 1987 et de 2001 sur le plan iconographique, le nombre de personnages féminins s'accroît avec le temps: neuf documents en 1987 et douze pour 2001. Mais il ne s'agit là que d'une augmentation relative, car la présence des personnages masculins s'est fortement accrue, passant dans la même période de vingt- 
sept à soixante-cinq. Par ailleurs, l'analyse qualitative du discours révèle une toute autre réalité.

L'ouvrage de 1987 montre la femme (sur un plan universel) dans des situations authentiques, exprimant la notion de liberté (sport, travail, loisirs, tenue vestimentaire), d'égalité dans la mixité ${ }^{16}$ (dans l'espace public et privé), et de responsabilité (implication dans la défense pour l'environnement et les droits de l'Homme). De nombreuses qualités telles que le courage, l'intelligence, l'éducation, le sérieux, l'instruction, ont été mises en exergue aussi bien chez la femme algérienne que chez la femme étrangère (pays anglophones du Sud). Les photographies de situations réelles et les dessins expriment des idées qui paraîtraient audacieuses (même si elles jouent sur l'ironie), et n'auraient pas de place dans un manuel destiné à un public d'aujourd'hui. ? Par exemple en page 112, en abordant les droits de la femme et la journée du 8 mars, le concepteur a introduit un dessin humoristique qui montre un homme en tablier, en train de laver la vaisselle, entouré de linge à laver, d'un aspirateur et d'une serpillière, et sa femme, assise sur un fauteuil, jambes allongées sur un repose-pied, lisant le journal en face de la télévision et déclarant : « Chéri, il faut bien l'admettre, nous ne serons jamais égaux. ». En page 133, en support à une photographie de femmes en voile et de femmes non voilées, est inséré un texte virulent de Nawel SAADAWI (écrit en 1966) sur l'origine du voile qui remonte au mythe d'Adam et Ève : la femme, considérée comme «source du mal et du péché » fut contrainte de couvrir son corps, ce que l'auteure analyse comme une atteinte aux « droits de l'Homme ${ }^{17}$. Pour résumer, la figure féminine dans ce manuel correspond à une image réelle, en adéquation avec le contexte de l'époque, et est valorisée par le concepteur qui a consacré tout un chapitre d'une trentaine de pages à la femme.

Pour ce qui est de l'ouvrage de 2001, mises à part deux photographies de l'ex-président des États-Unis d'Amérique, il contient exclusivement des dessins (souvent pris de l'Internet), de taille très réduite, conçus pour déclencher des activités d'apprentissage de la langue-instrument, et ne faisant que très rarement appel au vécu ou à la culture de référence de l'apprenant. Toutes les qualités attribuées à la femme par l'auteur du manuel de 1987, (affichées à travers des photographies très explicites, et de taille assez importante pour certaines qui prennent parfois toute une page), sont invisibles dans le livre de 2001 qui pourtant a été rédigé à la mémoire de l'autre auteur. On voit bien, à travers la comparaison de ces deux matériaux didactiques que la notion de visibilité ne garantit pas la valorisation du personnage.

Ce constat s'applique aux autres manuels.

Le manuel de 2000, confectionné dans l'urgence pour répondre à un manque criant de supports didactiques, ne comporte que des documents textuels, la plupart puisés dans d'anciennes éditions. Les rares textes présentent une image négative du personnage féminin; en effet dans les chansons telles que "Clementine», "Yesterday », "In the ghetto » et "Where have the flowers gone», la femme est associée au malheur: elle se noie dans la première chanson, elle quitte son ami éploré dans la deuxième, elle pleure son fils qui a été tué dans la troisième et elle perd son ami soldat dans la quatrième.

19 Dans le manuel de 2007, utilisé actuellement dans les classes de Terminale, l'image de la femme est soit surannée (symbolisée par des personnages de la mythologie grecque pour lesquelles les apprenants n'ont aucun référent dans leur contexte culturel), soit idéalisée (femmes jeunes, belles et surtout célèbres) ${ }^{18}$, mais rarement proche de la réalité. 
20 À travers ces quelques exemples, l'on découvre comment le message présent dans les textes officiels est en réalité traduit sous forme de discours didactique, puis pédagogique avant sa réception par les élèves. Afin d'étayer cette observation, nous avons procédé à un entretien et à des observations de séminaires pédagogiques avec l'un des concepteurs des manuels étudiés. Nous avons également tenu un journal de bord où sont notées toutes les informations concernant les manuels du secondaire, les impressions des enseignants, des élèves et de leurs parents, les questions débattues lors de journées d'études ou de colloques ou encore dans la presse ayant trait aux manuels, et mes propres réflexions. Tout ceci a contribué à vérifier le constat du traitement discriminatoire de la figure féminine dans les manuels scolaires qui va en s'accentuant avec le temps.

Quant à l'évaluation de ce travail, elle ne peut, pour le moment, se faire d'une façon satisfaisante, et ce pour des raisons d'archivage, de disponibilité de documentation, et de possibilité de sonder le personnel éducatif et autres acteurs sociaux impliqués dans cette situation ${ }^{19}$, et ne pourra se réaliser qu'après quelques années de recul par rapport à l'application de la réforme du système éducatif et l'étude parallèle du niveau académique de plusieurs cohortes d'étudiants dans les prochaines années pour mesurer l'impact de cette réforme sur leur apprentissage.

\section{Discussion}

Rendre invisibles les femmes semble lié à quelques traditions que certains veulent faire perdurer, mais que les femmes ont toujours tenté de transgresser. Comme l'écrit ACHERAR (p.40) : «Bravant les règles traditionnelles d'invisibilité, elles tissent au quotidien une nouvelle définition de la citoyenneté "au féminin", mais aussi d'une citoyenneté nouvelle pour tous ». C'est le cas dans les pays où la femme était auparavant reléguée au foyer, et aujourd'hui s'est imposée dans le domaine public. Malgré cela, leurs manuels scolaires continuent d'en véhiculer une image faussée (MOscoNI, p.169) allant jusqu'à propager la violence symbolique à travers leur contenu (RHIWI, 1999). La femme active, particulièrement, a mauvaise presse aussi bien dans la société que dans les manuels ${ }^{20}$. Pourtant le traitement discriminatoire de la figure féminine a maintes fois été dénoncé un peu partout, même si l'androcentrisme prévaut encore d'une façon inconsciente ( JUNOD, 2004, p.22), y compris chez les conceptrices de manuels ou autrices de contes pour enfants.

Le déni est une forme encore plus grave que la discrimination. C'est précisément cet aspect qui doit disparaître du paysage pédagogique car il n'est pas en conformité avec la réalité sociale. Les chiffres concernant la scolarisation des filles et ceux, pourtant incomplets, se référant à l'emploi féminin prouvent que la femme a occupé l'espace public en Algérie et participe activement à la vie du pays. Il semble logique et légitime qu'elle soit représentée de façon réaliste dans les ouvrages scolaires. D'autant que les textes officiels des programmes de l'éducation nationale recommandent d'éviter toute discrimination de quelque nature. La CNP est une institution officielle qui a pour mission de contrôler les contenus des manuels avant l'aval donné par le ministère de l'Éducation nationale. Comment expliquer que certains aspects négatifs puissent encore prévaloir dans ce domaine? Les conditions d'urgence ont été invoquées pour expliquer un grand nombre d'erreurs relevées dans certains manuels. Le problème ne concerne pas quelques coquilles qui se sont glissées çà et là et qui ont été tout naturellement corrigées par les 
enseignants eux-mêmes. La période difficile qu'a connue l'Algérie pendant plus d'une décennie est à l'origine de beaucoup de dysfonctionnements. L'école a été touchée de plein fouet par la violence terroriste ; les enseignantes, en particulier, ont payé un lourd tribut pendant cette période. Toute l'organisation du système éducatif a été bouleversée, incluant bien sûr, toute la chaîne du livre. Durant ces années, le mot d'ordre était « la débrouille » et ce n'est qu'en 2003, lorsque la nouvelle réforme du système éducatif fut mise en application et que de nouveaux programmes et manuels firent leur apparition que les choses ont commencé à changer. La recherche ne possède pas assez de recul pour évaluer la nature de ces changements mais de nombreux travaux menés actuellement nous renseigneront sur l'impact de la réforme. Toujours est-il que, pour le moment, il est difficile de croire le traitement de la figure féminine dans les manuels scolaires puisse changer d'un jour à l'autre. Les représentations sont fortement ancrées, entretenues et consolidées au sein de la société. L'enseignement est conçu, en grande partie, en référence à la nature des textes fondateurs qui ont inspiré le référentiel des programmes. C'est un fait connu que les nations désirent inculquer leurs valeurs à travers les programmes scolaires en vue de la formation de citoyens. Comme le rapporte MORIN (2004, p.82), soutenant la thèse de мАтнY (1996): «il n'y a pas d'enseignement idéologiquement neutre ". Dans certains cas, le poids de cette charge idéologique est très important, au point de se substituer à l'objectif de la formation éducative ${ }^{21}$. Au même moment, le message idéologique n'est pas explicite; il se réfugie derrière une terminologie qui correspond tout à fait au domaine qu'il infiltre, se transmet par conséquent, d'un niveau à l'autre dans le processus de formation curriculaire et se retrouve logiquement présent lors de la transposition didactique. Ce curriculum caché, ou idéologie diffuse (MOSCONI, p.24-25), qui, initié par les textes officiels et leur traduction en discours didactique, soutenu et renforcé par l'environnement culturel et l'adhésion de la majorité des acteurs du système éducatif (BECCHI, 1994 ; JACQUEMET, 2002 ; JUNOD, 2004 ; FONTANINI, 2007 ; FORQUIN,1985, 2006 ; GEORGESCU, 2005 ; PERRENOUD, 1986, 1993), est inculqué en même temps que le curriculum formel et peut avoir le même impact que celui-ci sur l'apprenant ${ }^{22}$. Les instances éducatives peuvent ainsi, par la route naturelle du processus curriculaire, transmettre les lignes essentielles d'un discours idéologique qui, transformé en discours didactique, semble tout à fait inoffensif, logique et évident.

\section{Conclusion}

Alors que le paysage social se transforme, pour que la situation s'améliore, il faudrait que les différents acteurs sociaux s'impliquent pour faire de l'école non pas un lieu de confrontations et d'enjeux idéologiques, mais un lieu de savoir, agréable et ouvert sur le monde. Que ce soit les praticiens, les didacticiens, les concepteurs de manuels, les décideurs ou les parents d'élèves, toutes ces personnes sont concernées par l'avenir de l'école.

Cet article a tenté de décrire une situation pédagogique aux sérieuses répercussions sur les utilisateurs-trices de ces manuels. À travers le livre scolaire, c'est tout un projet de vie que l'on inculque à l'élève, d'où l'importance du manuel (NAїT-BRAHIM, 2005), qui représente dans les sociétés traditionnelles, le seul accès au savoir, «le livre des livres » selon FONTANINI (2007). N'emploie-t-on pas en Algérie l'expression « je vais lire » pour dire « je vais à l'école »? 


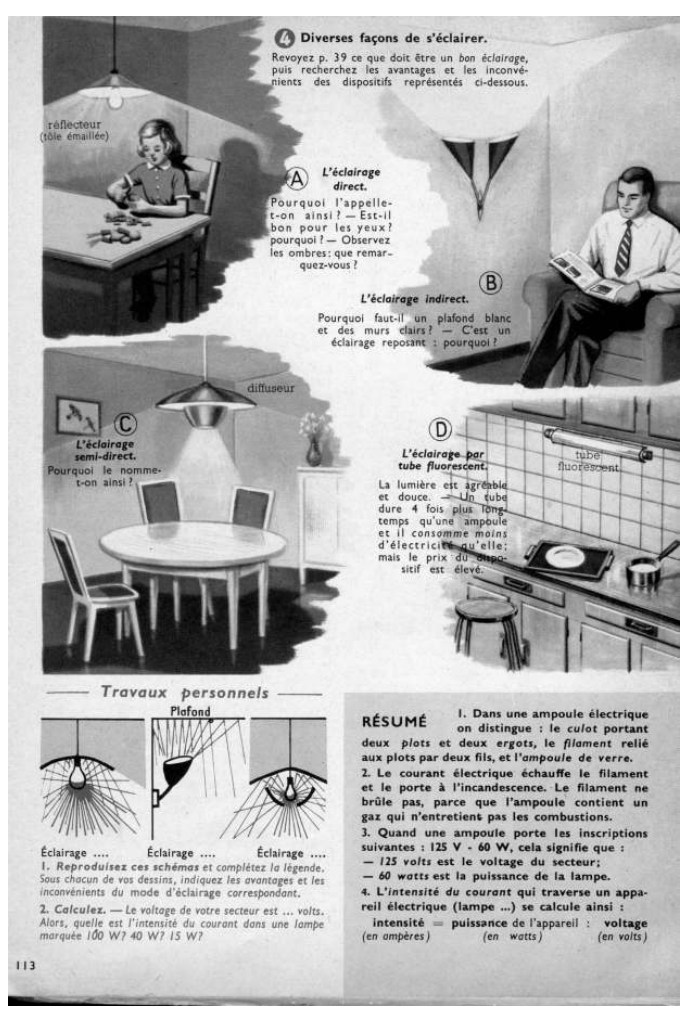

Sciences appliquées, classe de fin d'études, écoles urbaines de garçons, Marcel ORIEUX et Marcel EVERAERE, Paris : HACHETTE, 1958, 190 p., p. 113 - Source : CEDRHE.

\section{BIBLIOGRAPHIE}

ACHERAR L., Regards sur filles et femmes en Algérie in Les Cahiers Pédagogiques n³72, p.40-41, 1999 ACHOUR C. et MORSLY D., Entre un enracinement et un devenir. Le discours algérien sur la femme et la famille, in Plurial, publication du Celilif, Rennes, p.7-19, 1985

BECCHI, E., Le curriculum. D'un point de vue didactique à une perspective historique, in Histoire de l'Éducation, nº 61, janv. 1994, p.61-71 (trad. de l'italien par M.M. COMPÈre).

BOUGUERRA T., Apprentissage écodidactique des représentations de l'interculturalité dans les manuels algériens, in Cahiers de langue et littérature n5, OPU, Mostaganem, 2008

BRUGEILLES C., CROMER S., Promouvoir l'égalité entre les sexes par les manuels scolaires. Un guide pour les acteurs et actrices de la chaîne du livre. Paris, Unesco, 2008, 98 p. http://unesdoc.unesco.org/ images/0015/001588/158897f.pdf

BRYMAN, A., The debate about quantitative and qualitative research : a question of method or epistemology? in The British Journal of Sociology, vol. XXXV, ${ }^{\circ} 1,1981$, p.75-92.

CORDIER C., Les éléments constitutifs du manuel, in Revue de didactologie des langues-cultures, $\mathrm{n}^{\circ} 125$, 2002, p.25-36. 
DOBZHANSKY T., L'homme en évolution. Paris : Flammarion, 1966

FORQUIN J.C., L'approche sociologique des contenus et programmes d'enseignement, in Perspectives documentaires en sciences de l'éducation, $\mathrm{n}^{\circ} 5,1985$, p. 31-70.

FRY G., CHANTAVANICH S. et CHANTAVANICH A., Merging quantitative and qualitative research techniques : toward a new research paradigm, in Anthropology and education quaterly, vol. XII, $\mathrm{n}^{\circ} 2$, 1981, p.145-158.

JАСОВ E., Qualitative research traditions : A review, in Review of educational research, vol. 57/1, 1987, p.1-50.

KACI, T., La gestion du changement dans le système éducatif, in Insanyat $n^{\circ} 6$, vol.II/3, CRASC, Oran, 1998, p.1-18.

матну P., Les choix épistémologiques, les idéologies et les valeurs dans les manuels de biologie : production d'instruments d'analyse pour la formation des enseignants. Thèse de doctorat en sciences de l'éducation non publiée, Genève: Université de Genève, 1996 MORIN Ed., À propos des sept savoirs, Éditions Pleins feux, Mayenne, 2000 MORIN Em., Le pouvoir du manuel scolaire, in Cahiers du Cirade, 3, 2004, p.77-88.

MOSCoNi N., Effets et limites de la mixité scolaire, in Travail, genre et sociétés n¹1, 2004, p.165-174. MUCCHIELLI A., Le développement des méthodes qualitatives et l'approche constructiviste des phénomènes humains, in Recherches qualitatives Hors-série $\mathrm{n}^{\circ} 1$, 2005, p.7-40.

MUCCHIELLI R., L'analyse de contenu des documents et des communications (6e éd.) (Coll. « Formation permanente en sciences humaines ») Paris, les éditions ESF, 1988

NAIT-BRAHIM A., Don't Wake up a Sleeping Learner : The Soporific Substance of the Textbook, Actes du colloque « Le manuel dans tous ses états ", in Revue du LAROS n², éditions Dar el Gharb, Oran, 2005, p.80-99.

PERRENOUD P., Curriculum : le formel, le réel, le caché, in Houssaye (dir.) La pédagogie : une encyclopédie pour aujourd'hui, Paris, ESF, 1993, p.61-76.

PESHKIN A., The goodness of qualitative research, in Educational researcher, vol.22, n² 2, 1993, p.23-29.

PINARD R., POTVIN P., ROUSSEAU R., Le choix d'une approche méthodologique mixte de recherche en éducation, in Recherches qualitatives, vol.24, 2004, p.58-80.

RICHARD S., L'analyse de contenu pour la recherche en didactique de la littérature. Le traitement des données quantitatives pour une analyse qualitative : parcours d'une approche mixte, in Recherches qualitatives, vol.26 (1), 2006, p.181-207.

SADOU H., Scolarisation, travail et genre en Algérie, in Afrique et développement, vol. XXXII, $\mathrm{n}^{\circ} 3$, 2007, p.121-130.

VAN DeR MARREn J-M., Méthodes de recherche pour l'édition. (2e éd.) Montréal : Les Presses de l'Université de Montréal, 1996 


\section{Sitographie}

BOUсHоUсHA I. et LосоH T., Les manuels scolaires en Tunisie reflètent-ils la réalité tunisienne ?, Les numériques du CEPED, INED, Paris, 2008

http://www.ceped.org/cdrom/manuels_scolaires/sp/intro.html Consulté le 20 mai 2009.

BRUGEILLES C., CROMER S. et LOCOH T., Analyser les représentations sexuées dans les manuels, in Les numériques du CEPED, Paris, 2008

http://www.ceped.org/cdrom/manuels_scolaires/Consulté le 20 mai 2009.

FORQUIN J-C., Apprentissages, culture scolaire et problématique curriculaire, in Le blog des savoirs CDI, CNDP, 2006

http://www.cndp.fr/savoirscdi/index.php Consulté le 30 septembre 2010

GEORGESCU D., Le rôle de l'éducation dans les situations de post-conflit. Les expériences du Bureau International de l'Education (BIE), in M. Aligisakis (dir.) Europe et sortie des conflits, 2005, p. 87-102. http://www.unige.ch/ieug/publications/euryopa/Aligisakis3.pdf consulté le 30 septembre 2010. HERRERA P.G., Sexisme, école, éducation in Magazine 360 ${ }^{\circ}$, [En ligne], mis en ligne 2004. http://www.tarihvakfi.org.tr/dkih/download/gulsun_gunenli.pdf Consulté le 20 mai 2009.

JACQUEMET N., Genre et école, in Résonances n ${ }^{\circ} 8$, avril 2002, p.4-5,

http://vs.ch/Press/DS_314/Doc-200521-8562/Fr/02\%2520 Resonances, consulté le20 mai 2009.

JUNOD H., Le sexisme des manuels et documents scolaires utilisés au XXe siècle dans les cantons romands, in Work in Progress, 2001, p.22-25.[En ligne], mis en ligne 2004.

http://www.unil.ch/webdav/site/liege/shared/.../workinprog_LIEGE04.pdf consulté le 20 mai 2009

JUNOD H., Le sexisme dans les manuels scolaires, Entretien in F-Information-Genève, Dossiers Voi (x)es, 2004

http://www2.unil.ch/liege/Egalens/index.html consulté le 20 mai 2009

KHALED K., La charge du programme crée du désordre chez les élèves, in El Watan, 31 aôut 2009, p.5 [en ligne], mis en ligne le 30 aôut 2009,

www.berberes.com/ archives/index.php consulté le 30 septembre 2010.

KOURTA, D., Une égalité non acquise, in El Watan , 19 février 2007 [ en ligne],

www.algeria-watch.org/fr/ article/ femmes/femme_societe.htm Consulté le 20 mai 2009

OUESLATY B., MC ANDREW M. et HELLY D., Le traitement de l'Islam et des musulmans dans les manuels québécois de langue française, in Rapport Islam Québec, 2010

http.chereum.umontreal.ca/publications_pdf /Traitement\%20de\%20l'Islam.pdf consulté le 30 septembre 2010

PAIVANDI, S., L'individu dans les manuels scolaires en Iran, in Cemoti, $\mathrm{n}^{\circ} 26$ - L'individu en Turquie et en Iran, [En ligne], mis en ligne (non précisé),

http://cemoti.revues.org/document36.html Consulté le 20 mai 2009.

PERRENOUD, P., Vers une lecture sociologique de la transposition didactique, 1986

http://www.atelierdephilosophie.ch/.../transposition_didactique_perrenoud.pdf

RHIWI, L., L'image de la femme et les violences symboliques à son égard au Maroc, in Collectif 95

MaghrebEgalité, 1999

http//www.retelilith.it./ee/host/maghreb/htm/magh.htm consulté le 20 mai 2009. 


\section{Corpus de manuels}

ARAB S.A., RICHE, B. et BENSEMMANE, M., New prospects. secondary education, year three. Alger :

O.N.P.S., 2007, 223 p.

BenZian A., BOUAKKaZ H. et HadjIDJ B. S., Comet, 3 A.S., Alger : O.N.P.S., 2000, 143 p.

BEREKSI K.E.S., My Book of English 9, 3 as all streams, Alger : O.N.P.S., 2001, 280 p.

LOUZNADJI M., English, open doors, 3e A.S., Oran : EDIK, 2003, 199 p.

MENASSERI H., ASSELAH Y., HAMDAD N., HENICHE F., KADI R. et TAHARBOUCHET S., Think it over, Alger :

I.P.N., 1987, 223 p.

\section{NOTES}

1. Le 13 mai 2000, le président Bouteflika installait officiellement la Commission Nationale de la Réforme du Système Éducatif (CNRSE). Le rapport de la réforme fut rendu public en 2003. Durant la décennie qui a précédé l'application de cette réforme, la presse algérienne s'est fait l'écho d'interminables débats autour de l'école auxquels ont participé de nombreux universitaires, des journalistes, des membres du secteur de l'éducation ainsi que des parents d'élèves.

2. Les ouvrages précédents n'avaient pas connu de réels changements depuis l'instauration de l'École fondamentale à la fin des années 1970 (Cf. les quotidiens El Watan des 3/1/00 et 20-21/4/01 et La Voix de l'Oranie du 20/9/01).

3. Allocution du Président de la république lors de l'installation de la CNRSE (cf. note 1).

4. Conclusion d'un sondage effectué par l'institut Echotechnics pour El Watan et publié dans ce quotidien les 7 et 8 octobre 2009.

5. Un premier ouvrage concernant l'Anglais écrit a déjà vu le jour en 2006 ; un manuel de lecture critique est en cours de rédaction.

6. Une étude synchronique n'aurait pas été aussi significative du fait que la plupart des manuels en usage dans les différents paliers de l'enseignement secondaire et moyen sont confectionnés par les mêmes équipes désignées à la suite de la nouvelle réforme du système éducatif.

7. Quelques universitaires ont effleuré le problème en étudiant au cours des années 1980 des manuels de français (cf. ACHOUR et MORSLY, 1985), mais nous n'avons rencontré aucune étude scientifique publiée.

8. PINARD et al. (2004, p.60) soulignent qu'«une approche de recherche traduit une manière d'être et de faire qui est en accord avec ce que nous estimons être juste dans les rapports que nous entretenons avec le réel.»

9. «...l'association de techniques qualitatives et quantitatives au sein d'une même étude ou d'un même projet...semblerait offrir d'excellentes possibilités pour développer une compréhension plus approfondie des processus pédagogiques.»

10. Durant l'entretien avec l'auteur, celui-ci m'a affirmé que de nombreux enseignants l'utilisaient dans leurs classes, surtout pendant la période pré-réforme qui se caractérisait par un manque crucial de manuels.

11. Comme l'affirme MORIN (2000, p.26) «C'est donc toujours la contextualisation qui permet d'avoir une connaissance pertinente».

12. Les manuels scolaires n'avaient pas connu de réaménagement significatif depuis une trentaine d'années. Le manuel de 1987 est basé sur un «état de besoins», celui de 2000, rédigé dans l'urgence, ne comporte pas de partie iconographique et celui de 2001fut conçu dans le cadre 
de l'introduction de l'Anglais au primaire. Le manuel de 2003 a tenté de répondre à de nouveaux besoins et finalement, celui de 2007 est basé sur les programmes de la nouvelle réforme.

13. CORDIER (2002) distingue trois types de textes formant le corps central d'un manuel de langue : les textes exemplificateurs (ou énoncés-modèles) comportant les éléments linguistiques à enseigner, les textes analytiques qui décomposent la langue en faisant intervenir concepts et opérations métalinguistiques, et les textes déclencheurs (exercices, activités, tâches).

14. L'utilisation de la réalité de l'Autre comme un élément de comparaison avec celle des apprenants aurait certainement été très intéressante sur un plan pédagogique.

15. Cf. le référentiel des programmes établi suite à la réforme du système éducatif en 2005.

16. MOSCONI (2004, p.171-2) note que «la mixité n'est pas un principe suffisant, si elle ne s'accompagne pas d'une véritable politique d'égalité».

17. ACHERAR (1999, p.41) écrit à ce propos: «La volonté de mettre les femmes à l'écart, de les renvoyer à l'invisibilité se fonde, tant à l'école que dans la société, sur une démarche qui prend le corps des femmes comme enjeu politique.».

18. RHIWI (1999) note à propos de la femme marocaine: «La femme idéalisée dans les manuels scolaires est aussi celle qui reste jeune et ne fait jamais preuve de lassitude [...]Les qualités intellectuelles sont occultées, et à mesure qu'elle avance en âge, les stéréotypes s'apparentent plutôt à l'injure».

19. Cf. KACI (1998).

20. KoURTA (El Watan, 19 février 2007) souligne : «La femme militante et active est mal vue au sein de la société. Une idée entretenue aussi bien par les médias lourds (émissions télévisuelles) que les programmes enseignés à l'école algérienne. Ces messages véhiculés agissent sur une réalité en orientant les représentations et les comportements. Il y a une régression dans le discours et le discours religieux extrémiste justifie ainsi la violence et la marginalisation de la femme».

21. Cf. BOUGUeRRA (2008), KHALED (2009).

22. Cet aspect mériterait l'intérêt des chercheurs car il pourrait éclairer bon nombre de problèmes qui se posent aux didacticiens et aux praticiens concernant la question de l'apprentissage.

\section{RÉSUMÉS}

En l'espace de deux décennies (1987-2007), la représentation du personnage féminin dans les manuels d'Anglais algériens destinés à la classe de 3e année secondaire (Terminale) s'est considérablement réduite, ne reflétant ni la réalité sociale, ni les objectifs de la réforme du système éducatif. Quels sont les facteurs qui ont engendré ce phénomène ? Peut-on parler d'un « curriculum caché » dans les orientations pédagogiques? Quel impact celui-ci peut-il avoir sur l'apprentissage ? Ces questions ont été abordées dans le cadre d'une recherche-action, fondée sur une approche méthodologique mixte, ayant pour objectif principal le changement.

The representation of the feminine character in Algerian textbooks of English aimed at the 3rd year secondary class has significantly decreased over the last two decades (1987-2007) thus, neither reflecting the social reality, nor the objectives of the educational system reform. What are the reasons behind such a phenomenon? Can we speak of a "hidden curriculum" in the pedagogical orientations? Which impact can the former have on learning? Such inquiries will be tackled in the following study, undertaken in the context of a research-action and based on a 
methodological approach combining quantitative and qualitative analysis, essentially directed towards change.

INDEX

Mots-clés : genre, manuel scolaire, recherche-action, représentation

Keywords : gender, representation, research-action, textbook

\section{AUTEUR}

FÉRIDA LAKHDAR-BARKA

Université d'Oran Es-Sénia, Algérie 